\title{
Advantages of the local electrode for atom probe tomography applications.
}

\author{
R. Ulfig*, D. J. Larson*, T. F. Kelly* \\ * Imago Scientific Instruments Corp. 6300 Enterprise Lane, Suite 100, Madison, WI 53719
}

New developments in atom probe tomography, namely the implementation and improvement of the local electrode ${ }^{\mathrm{TM}}$, have enabled new applications and capabilities. Historically atom probe tomography had been limited in scope by the difficult and time consuming art of sample preparation, the time required to collect data over the region of interest, and the inherent limitations of conducting time consuming multi-variant experiments to optimize the quality of the data collected, but this is no longer the case.

The local electrode's primary hardware advantage is due to the electric field enhancement of the proximity of a counter electrode to the specimen tip. This proximity effects a reduction of the geometrical coefficient, $\mathrm{K}$-effective $\mathrm{F}=\mathrm{V} /\left(\mathrm{K}_{\text {eff }}{ }^{*} \mathrm{r}_{\text {specimen }}\right)$, by approximately by a factor of $2.5[1]$. This field enhancement allows the use of lower pulse voltages[2,3]. The lower pulse voltage required enables higher speed, and narrower width voltage pulses. The benefit of the local electrode is in the ability to have high mass resolution and high field of view with large collected volumes possible in a short amount of time.

The ability to analyze larger volumes in shorter periods of time affords great advantages, but specimen preparation remains one of the most important experimental aspects of atom probe tomography[4]. Without a local electrode all atom probe specimens are limited to a solitary needle geometry to provide a sufficient electric field for field ionization to remove the atoms from the surface of the specimen. For bulk metals with a well established electrochemical etching chemistry this is a straightforward process, but for most other material classes the process can be complex, time consuming and expensive. Even for a simple deviation from the most simple sample type, grain boundary analysis from a metal for example, one must not only have an etch capability that does not damage the grain boundary preferentially, but iterative TEM inspection steps must be performed to locate the region of interest near enough the apex of the sample to allow field analysis through the region of interest prior to the sample blunting or fracturing.

Leveraging well developed specimen preparation techniques for TEM and the advantage of local field enhancement provided by the local electrode, novel sample preparation geometries referred to as microtips have been demonstrated that have not only simplified the atom probe specimen preparation and analysis, but have enabled new applications as well as straightforward methods of doing direct comparisons of TEM and atom probe tomography data on the same specimen. The wide field of view provided by a local electrode instrument such as the LEAP $3000^{\mathrm{TM}}$ simplifies sample preparation further as the requirements for the placement of the region of interest at the apex of the sharpened tip are reduced by a factor of 5 or more over the tip of the specimen due to the wider field of view and large collection volumes enabled by the high rate of data collection. In addition, microtip arrays which dramatically speed both specimen preparation and analysis, for the first time ever, allow large scale design-of-experiment investigations that were previously prohibitively time consuming.

[1] T.F. Kelly et. al., Microscopy and Microanalysis 10 (2004) 
[2] O. Nishikawa and M. Kimoto, Appl. Surf. Sci. 76/77 (1994)

[3] T. F. Kelly, P.P. Camus, D.J. Larson, L.M. Olzmann, and S.S. Bajikar, Ultramicroscopy (1995)

[4] M.K. Miller, G.D.W. Smith, Atom Probe Microanalysis, MRS materials Research (1989)

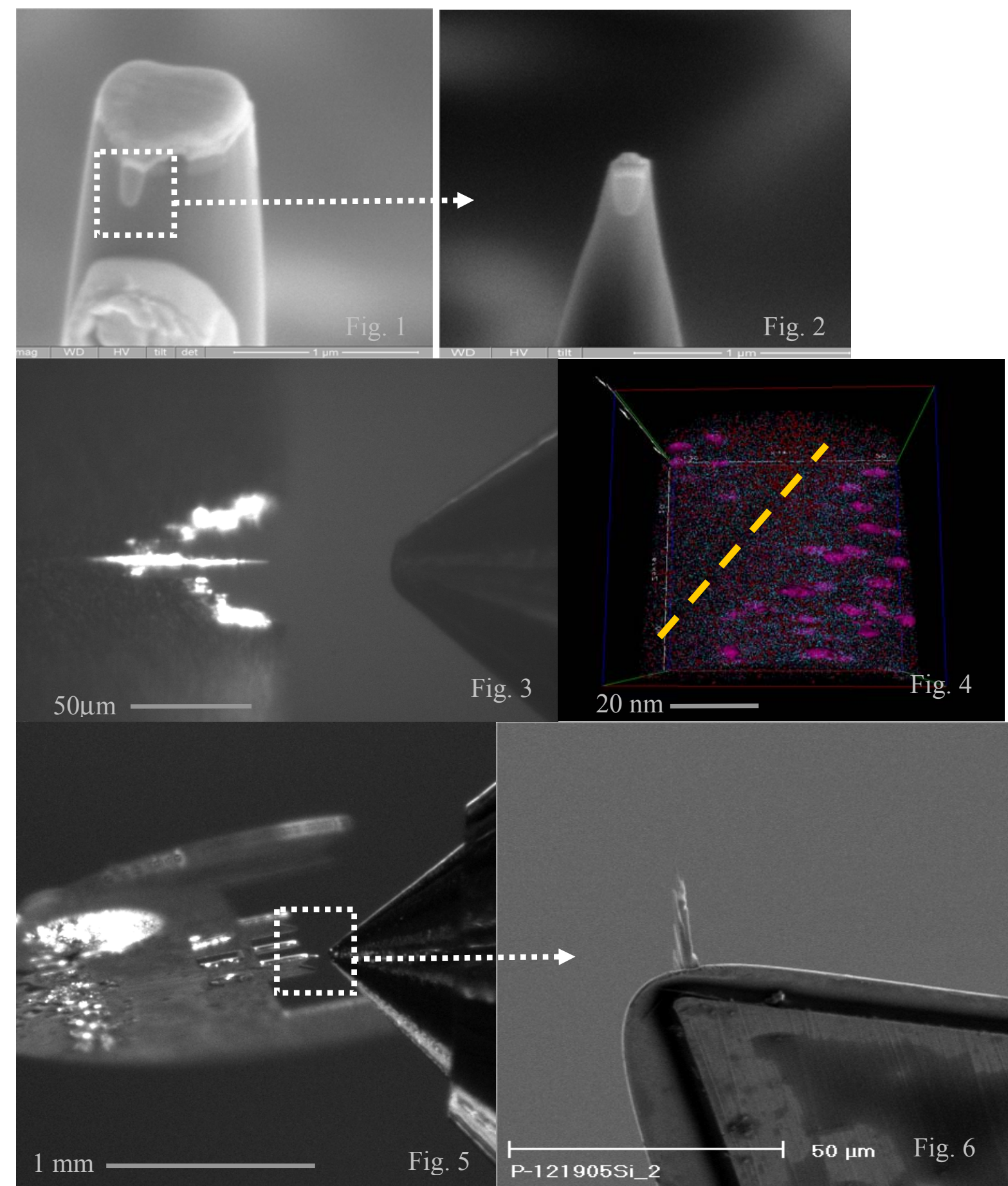

Fig. [1/2]. Site specific sample preparation of a transistor junction lifted out of a wafer and mounted to a microtip substrate. Fig. [3/4]. Site specific prepared sample adjacent to the local electrode during analysis and the resultant data showing the targeted grain boundary. Fig. [5/6]. Site specific lift-out of a dielectric to a standard TEM grid and adjacent to the local electrode during analysis 